\title{
HYPOTHERMIE ACCIDENTELLE: TRAITEMENT D'UN CAS PAR IRRIGATION PERITONEALE
}

\author{
Henri Desmeules et Conrad Blais
}

L'HYPOTHERMIE ACCIDENTELLE est un incident qui se produit surtout dans les régions nordiques, quoique cette condition n'est pas exclusive aux régions où le climat est rigoureux. Parmi les facteurs qui favorisent l'apparition de l'hypothermie accidentelle, citons l'intoxication alcoolique ou médicamenteuse, les accidents de la route, les noyades, l'exposition aux éléments et l'épuisement survenant particulièrement lors de randonnée en forêt. ${ }^{1.2}$ Chez le vieillard, l'hypothermie accidentelle est associée aux maladies mentales, à l'hypothyroïdie, à l'insuffisance surrénalienne, à l'hypopituitarisme et aux maladies cardio-vasculaires. ${ }^{3}$

En 1966, le rapport du "Committee on Accidental Hypothermia of the Royal College of Physicians" a rapporté une mortalité de 37.3 pour cent de tous les patients admis en hypothermie et une mortalité de 73.3 pour cent chez les patients dont la température était inférieure à $85^{\circ} \mathrm{F}$ $\left(29.4^{\circ} \mathrm{C}\right){ }^{4}$ Mème s'il semble que le pronostic se soit amélioré au cours des dernières années, ta mortalité demeure élevée et un traitement inten. sif s'impose.

Le traitement a varié au cours des années. allant du simple réchauffement passif à la température ambiante à un réchauffement plus énergique par voie externe (couvertures électriques, matelas chauffants, bains chauds) ou par voie interne (lavage d'estomac et lavements chauds, ventilation avec des gaz préchauffés, circulation extracorporelle et irrigation péritonéale). Nous rapportons dans ce travail un cas d'hypothermie accidentelle profonde traité par irrigation péritonéale.

\section{Presentation du cas}

Un patient mâle de 67 ans, alcoolique, a été

${ }^{*}$ Henri Desmeules, M.D., F.R.C.P.(C), Profes seur agrégé. Département d'Anesthésie-Réanimation, Université Laval. Membre du Département d'Anesthésie-Réanimation du Centre Hospitalier de l'Université Laval, 2705 Bd. Laurier, Québec (Québec). GIV $4 G 2$. Conrad Blais, M.D., Résident, Département d'Anesthésié-Réanimation, Université Laval. Actuellement membre du Département d'Anesthésie-Réanimation de l'Hôpital St-joseph de Rimouski, 150 rue Rouleau, Rimous découvert inconscient. un matin de janvier. couché dans la neige à l'entrée de sa demeure. A ce moment la température extérieure était de $-22^{\circ} \mathrm{C}$. Lors de son admission à la salle d'urgence, on a constaté une rigidité musculaire très importante; la peau était froide, présentait des lividités et il n'y avait aucune réponse aux stimuli. Le pouls et la tension artérielle étaient imperceptibles. La respiration étaient lente, très superficielte et la température rectale au moment de l'admission était de $26^{\circ} \mathrm{C}$.

Peu après son admission, le patient été transféré à l'Unité des Soins Intensifs où il a été intubé par voie nasotrachéale $\left(\mathrm{Fl}_{\mathrm{O}_{2}} \mathbf{0 . 4}\right)$. A ce moment, la température rectale mesurée par un thermocouple électronique était de $25^{\circ} \mathrm{C}$. Ce niveau de température a été confirmé à l'aide d'un thermomètre à mercure permettant la lecture de basses températures. Les paramètres usuels ont été monitorés: pouls, pression artérielle, ẻlectrocardiogramme et respiration. Au moment de l'admission et en l'absence d'assistance ventilatoire, la mesure des gaz artériels après correc. tion en fonction de la température du patient ${ }^{5}$ a donné les résultats suivants: $\mathrm{H}^{+} 55 \mathrm{nmol} / \mathrm{litre}(\mathrm{pH}$ 7.26). $\mathrm{Pa}_{\mathrm{CO}_{2}} 3.59 \mathrm{kPa}$ (27 Torr) $\mathrm{Pa}_{\mathrm{O}_{2}} 10.64 \mathrm{kPa}$ (80 Torr) $\left(\mathrm{FI}_{\mathrm{Oz}} 0.21\right), \mathrm{HCO}_{3}-13 \mathrm{nmol} / \mathrm{litse}$. L'alcoolémie était de $0.26 \mathrm{~g}$ pour cent. L'électrocardiogramme a montré une bradycardie sinusale variant entre 50 et $60 / \mathrm{min}$. Cependant l'interprétation de l'électrocardiogramme s'est avérée très difficile en raison des artéfacts engendrés par des tremblements musculaires fins que présentait le patient.

Dans un premier temps, le patient a été placé sur un matelas chauffant de type Gaymar réglé à $35^{\circ} \mathrm{C}$. Cependant, en raison de l'importance de l'hypothermie, il a été décidé d'utiliser une technique de réchauffement interne et une irrigation péritonéale a été commencée. Un cathéter péritonéal a été mis en place et une solution de Dianeal 1.5 pour cent, prếchauffée à $37^{\circ} \mathrm{C}$, a servi à irriguer la cavité péritonéale au rythme $2,000 \mathrm{ml} / \mathrm{h}$. Un réchauffeur de sang Fenwal à chaleur sèche (4R4304) a été utilisé pour maintenir la température de la solution d'irrigation à $37^{\circ} \mathrm{C}$ et un "manchon pneumatique" a servi à 


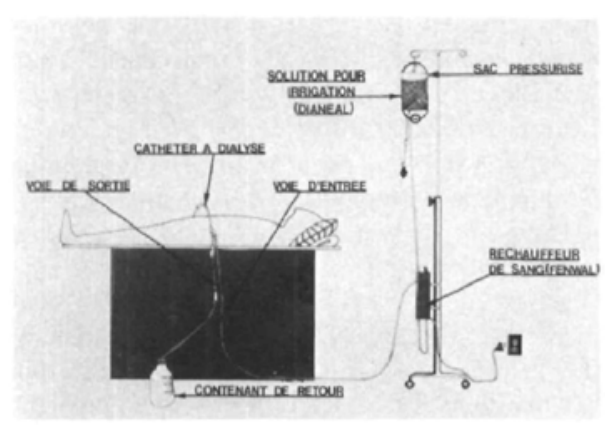

Floure 1 Montage utilisé pour lirrigation péritonéale.

maintenir la pression nécessaire autour de la solution de Dianéal pour obtenir le débit d'injection nécessaire (Figure 1).

Dans les minutes qui ont suivi la mise en marche de l'irrigation péritonéale, on a pu observer une élévation rapide de la température du patient, élévation qui s'est poursuivie au cours de toute la période d'irrigation qui a duré trois heures. La figure 2 illustre l'évolution de la température du patient au cours de la période d'irrigation péritonéale.

Le patient a repris conscience lorsque la température a atteint $30^{\circ} \mathrm{C}$; les signes vitaux se sont progressivement normalisés et le patient a pu être extubé huit heures après son admission. L'acidose métabolique s'est corrigée au cours du réchauffement sans addition d'alcalin.

Le patient a évolué favorablement et a été libéré après dix jours d'hospitalisation. Au départ, ainsi qu'un mois après avoir été libéré de l'hôpital, un examen de contrôle n'a pu mettre en évidence aucune séquelle importante.

\section{Discussion}

En 1961, Duguid' a décrit trois degrếs d'hypothermie selon la température rectale: l'hypothermie légère $\left(36^{\circ} \mathrm{C}\right.$ à $\left.32.3^{\circ} \mathrm{C}\right)$, modérée $\left(32.3^{\circ} \mathrm{C}\right.$ à $\left.23.9^{\circ} \mathrm{C}\right)$ et sévère $\left(23.9^{\circ} \mathrm{C}\right.$ et moins). Cette classification correspond aux diverses réponses de l'organisme au froid.

Lors d'une hypothermie légère, l'organisme lutte pour maintenir son homéostasie: le pouls d'accélère, la respiration est rapide et la tension artérielle s'élève suite à une libération de catécholamines. Il y a une vaso-constriction périphérique et la circulation est redistribuée vers les organes vitaux. Une des réponses physiologiques qui visent à rétablir la température centrale est la production de frisson.
EVOUION OE LA TEMFERATUFE RECTALE

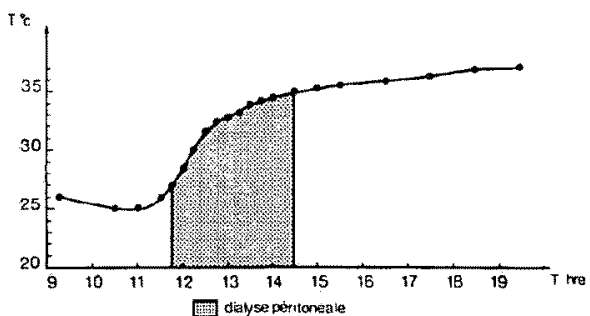

Figure 2 Evolution de la température du patient au cours de l'irrigation péritonéale.

A partir de $32.3^{\circ} \mathrm{C}$, on assiste à un épuisement graduel des mécanismes de défenses de l'organisme. Le frisson est aboli, le pouls et la respiration ralentissent et on constate une chute de la tension artérielle. Au-dessous de $28^{\circ} \mathrm{C}$, le rythme cardiaque devient irrégulier et une fibrillation ventriculaire peut apparaitre à tout moment.

Il existe une relation directe entre l'irritabilité du myocarde et la profondeur de l'hypothermie. Le plus souvent, la fibrillation ventriculaire apparaît lorsque la température centrale se situe entre $28^{\circ} \mathrm{C}$ et $25^{\circ} \mathrm{C} .6 .7$

Cette tendance à la fibrillation est attribuable à divers facteurs dont: l'hypoxie, les déséquilibres acido-basiques, la stimulation au moment de l'intubation et autres manipulations du patient hypothermique. ${ }^{8}$ Par contre, certaines études animales suggèrent que l'alcool éthylique aurait un effet protecteur contre la fibrillation venfriculaire chez le patient hypothermique. ${ }^{9}$ Lloyd et Mitchell: ${ }^{\circ}$ ont propose une hypothèse selon laquelle la fibrillation ventriculaire survient au cours de l'hypothermie lorsque la conduction êlectrique intra-cardiaque se fait par le tissu musculaire plutôt que par les voies neuromusculaires normales. En hypothermie, la fibrillation ventriculaire n'est habituellement réversible que lorsque la température centrale est de $2^{\circ} \mathrm{C}$ plus élevée que la température à laquelle le coeur a fibrillé. ${ }^{7}$

Si la température continue à s'abaisser, il y a au-dessous de $24^{\circ} \mathrm{C}$, abolition complète de tous les mécanismes de défense. Les centres thermorégulateurs sont rendus inopérants et l'organisme perd sa chaleur comme un objet inanimé. C'est donc un point critique et tout abaissement au-delà de cette température, si ce n'est dans des conditions strictement contrôlées, comporte des risques sérieux pour la survie du malade.

Le traitement de l'hypothermie accidentelle doit être adapté au niveau de cette hypothermie. 
Ainsi on s'accorde pour dire que le meilleur traitement de l'hypothermie légère (au-dessus de $32.3^{\circ} \mathrm{C}$ ) demeure le rechauffement passif, $"$ c'est-à-dire de laisser le malade se réchauffer par lui-même à une température ambiante élevée. $\mathrm{Si}$ on procède ainsi, les problèmes et les complications sont pratiquement inexistants.

A une température inférieure à $32.3^{\circ} \mathrm{C}$, il faut utiliser des moyens beaucoup plus efficaces. Le réchauffement actif externe, d'abord considéré comme seule méthode valable. est maintenant abandonné pour plusieurs raisons, ${ }^{7.8 .11 .12}$ Cette forme de réchauffement est anti-physiologique car elle augmente les besoins métaboliques en périphérie au moment où le cceurur, déprimé par le froid. ne peut répondre à cette demande. De plus. le réchauffement des extrémités amène une abolition précoce de la vasoconstriction, ce qui conduit à une stagnation du sang en périphérie et à une diminution du retour veineux. Nous avons donc un système cardiovasculaire fortement déprimé accompagné d'un "pooling" de sang au niveau des extrémités, ce qui pourrait très bien expliquer le choc du réchauffement décrit par plusieurs auteurs ${ }^{1.2}$ qui ont utilisé cette forme de traitement.

Par contre, le réchauffement actif interne amène beaucoup moins de complication et surtout il a démontré une efficacité certaine. ${ }^{7.8 .11 .12}$ En effet. plusieurs témoignages font état de résultats inespérés. Cette technique fait appel à des moyens dont la disponibilité varie d'un hòpital à l'autre.

La circulation extracorporelle offe les avantages les plus importants: elle permet de contrôler à la fois la température. la respiration et la circulation. C'est la méthode de choix lorsque le patient hypothermique est en arrêt cardiaque. Toutefois, elle comporte aussi l'inconvénient de ne pas être disponible dans tous les hôpitaux, et particulièrement dans les hôpitaux qui sont situés hors des grands centres urbains.

Si on exclut le lavage d'estomac et les lavements chauds ainsi que la ventilation avec des gaz pré-chauffés qui sont des méthodes peu efficaces, le seul moyen facile et disponible demeure l'irrigation péritonéale. ${ }^{712.14 .15}$ Cette méthode n'est pas exempte de complication, ${ }^{15,16}$ mais elle possède de nombreux avantages. Elle est relativement simple, facile à utiliser, disponible partout et ne requiert aucun équipement dispendieux. N'importe quel médecin peut utiliser cette technique en cas de besoin.

Il n'est pas absolument nécessaire d'utiliser des solutions spéciales pour irrigation périto- néale. En effet, Pickering ${ }^{7}$ suggère que des solutions isosmolaires telles que l'alternance de lactate Ringer avec du dextrose 5 pour cent dans l'eau pourraient ètre employées. Pour réchauffer ces solutions, on peut utiliser la technique habituellement employée pour réchauffer le sang.

Du point de vue physiologique. l'irrigation péritonéale fournit de la chaleur d'abord aux organes vitaux. Le creur est réchauffé rapidement et peut ainsi répondre aux demandes métaboliques accrues. La vasoconstriction périphérique décroit lentement et le volume circulant demeure adéquat. ce qui diminue le risque de choc du réchauffement. La recirculation de sang périphérique refroidi se trouve donc réduite au minimum.

Le cas présenté ici démontre qu'une hypothermie accidentelle peut étre traitée de façon rapide et efficace sans utilisation d'équipement sophistiqué. Quelques cas semblables ont été déjà rapportés dans la littérature avec le méme succès. ${ }^{7.14 .15}$ Lorsque la circulation extracorporelle n'est pas disponible et que le patient $n$ 'est pas en arrét cardiaque. l'irrigation péritonéale semble donc ètre la méthode de choix pour traiter les hypothermies modérées ou sévères.

\section{RÉSUMÉ}

Nous présentons un cas d'hypothermie accidentelle qui a subi un réchauffement actif interne grâce à l'utilisation d'une irrigation péritoneale. Chez ce patient, le réchauffement s'est produit rapidement et sans complication: le patient a pu quitter l'hôpital sans séquelle.

L'irrigation péritonéale constitue une méthode de réchauffement rapide et efficace qui peut être utilisée dans les cas d'hypothermie modérée ou sévère. Un autre avantage de cette méthode est qu'elle ne nécessite pas d'équipement compliqué et qu'elle peut ètre utilisée dans presque tous les centres hospitaliers, même dans les hôpitaux régionau $x$.

\section{SUMMARY}

A case of accidental hypothermia is reported. This patient was rewarmed by using a peritoneal irrigation. Rewarming was rapid and without complication. The patient was discharged from the hospital free from any sequellae. Peritoneal irrigation constitutes a fast and effective technique of core body rewarming in cases of moderate or severe hypothermia. Another advantage of 
this method is that it can be accomplished without complicated equipment and that it can be used even in small community hospitals.

\section{BIBLIOGRAPHIE}

1. REES, J.R. Accidental hypothermia. Lancet $t$ : $556-559$ (1958).

2. Duguid, H., Simpson, R.G. S STOWERS, J.M. Accidental hypothermia. Lancet 2: 1213-1219 (1961).

3 Bristow. G.. SMith. R.. Lee, J., Auty, A. \& TWEeD, W.A. Resuscitation from cardiopulmonary arrest during accidental hypothermia due to exhaustion and exposure. C.M.A. Journal: $1 / 7$ : 247-249 (1977)

4. Matthews, L. Postgrad. Med. J. 43: 662 667 (1967). (Report of the Committee on Accidental Hypothermia of the Royal College of Physicians) London (1966).

5. Severinghaus, J.W. Blood gas concentration in Handbook of Physiology. section 3: Respiration, Vol. 2, FenN, W.D., RAhN, H., (Eds.) Washington, Ann. Physiol. Soc. pp 1475-1487 (1965).

6. BLAIR. E. A physiologic classification of clinical hypothermia. Recent advances in surgery. 58: no. 3, 607-618 (1965).

7. Pickering, B.G., Bristow, G.K. \& Craig, D.B. Core rewarming by peritoneal irrigation in accidental hypothermia with cardiac arrest. Anesth. and Analg. 56: 574-577 (1977).
8. Wicktrom, P., Rutz, E., Lina. G.R.. HinterKOFF, J.P. \& HAGEIN, J.J. Accidental hypothermit. Core rewarming with partial bypass. Am. J. Surg. 13I: 622-625 (1976).

9. Fernandez. J.P.. O'Rourke. R.A. \& EwY.G.A Rapid active external rewarming in accidental hypothermia. J.A.M.A. 212: 153-156 (1970)

10. LLOYD. E.LL. \& MitCHELL. B. Factors affecting onset of ventricular fibrillation in hypothermia. Lancet 2: 1294-1296 (1974).

II. TRUSCOTT, D.G. WHITMER, B.F. \& CI.EIN, L.J. Accidental profound hypothermia. Successful resuscitation by core rewarming and assisted circulation. Arch. Surg. 106: 216-218 (1973).

12. SoUNG. L.S. Treatment of accidental hypothermia with peritoneal dialysis. C.M.A.J. 117:1415-1416 (1977).

13. Kugelberg, J., Schuller, H. Berg, N. \& KolLUM, B. Treatment of accidental hypothermia. Scand. J. Thor. Cardiovase. Surg. 1: 142-146 (1967).

14. Lash, R.F. Burdetre, J.A. \& Ozonl, T. Accidental profound hypothermia and barbiturate intoxication. J.A.M.A., 201: 269-270 (1967).

15. Patton, J.F. \& DoolitTle, W.H. Core rewarming by peritoneal dialysis following induced hypothermia in dogs. J. Appl. Physiol. 33: 800-804 (1972).

16. Miller. R.B. \& Tassistro. C.R. Peritoneal dialysis. N. Eng. J. Med. 281: 945 (1969).

17. Vanmonde, C.A. \& Perez. G.O. Peritoneal dialysis today. Kidney 10: 31 (1977). 